\title{
INTERVALO DE CORTE NA PRODUÇÃO DE MASSA SECA E COMPOSIÇÃo QUÍMICO-BROMATOLÓGICA DA Brachiaria brizantha cv. MG-5'
}

\author{
Effect of cutting periods on Brachiaria brizantha cv. MG-5 dry mass production \\ and bromatologic-chemical composition
}

\author{
Kátia Aparecida de Pinho Costa ${ }^{2}$, Itamar Pereira de Oliveira ${ }^{3}$, Valdemar Faquin 4 , Belmiro Pereira das Neves 3 , \\ Cristiane Rodrigues ${ }^{5}$, Fabrício de Menezes Telo Sampaio ${ }^{6}$
}

\begin{abstract}
RESUMO
O experimento foi conduzido em casa de vegetação, na Embrapa Arroz e Feijão, com o objetivo de avaliar o intervalo de corte na produção de massa seca, altura de planta e composição químico-bromatológica da Brachiaria brizantha cv. MG-5. O delineamento utilizado foi o inteiramente casualizado. Os tratamentos constituíram de quatro intervalos de corte (15, 20, 30 e 60 dias), com cinco repetições, totalizando 20 parcelas. Foram aplicados $100 \mathrm{mg} \mathrm{dm}^{-3}$ de nitrogênio, utilizando como fonte o sulfato de amônio, em todas as parcelas. A adubação nitrogenada foi parcelada, de acordo com os cortes de avaliações. Foram realizados quatro cortes para o intervalo de 15 dias, três cortes para o de 20 dias, dois cortes para o de 30 dias e um corte para o de 60 dias. A forrageira foi cortada a uma altura de $5 \mathrm{~cm}$ do solo. O aumento no intervalo de corte na Brachiaria brizantha cv. M-5 determina acréscimos na produção de massa seca e teores de FDN e FDA. Contudo, o inverso pode ser observado no teor de PB e concentrações de $\mathrm{P}, \mathrm{Cu}$ e Fe.
\end{abstract}

Termos para indexação: Absorção de nutrientes, valor nutritivo e freqüência de cortes.

\section{ABSTRACT}

The experiment was carried out in a green house, at Embrapa Arroz e Feijão. It aimed to evaluate the offect of cutting period on dry mass production and bromatologic- chemical composition of Brachiaria brizantha cv. MG-5. The experimental design was completely randomized, with five replications. The treatments were constituted by four cutting intervals (15, 20, 30 and 60 days). After cuttings at a distant from soil, of ammonium sulfate $\left(100 \mathrm{mg} \mathrm{dm}^{-3}\right)$ was applied to all treatments. Four cuttings were carried out at 15 - day intervals, three at 20 day-intervals, two at 30 - day intervals and one at a 60 day-interval. Increases in cutting intervals caused dry mass production, neutral detergent fiber and acid detergent fiber rase. However, the opposite was observed for bromatologic composition and phosphorus, copper and iron concentrations.

Index terms: Cutting frequency, nutrient absorption, nutrient value.

\section{(Recebido em 16 de maio de 2006 e aprovado em 22 de dezembro de 2006)}

\section{INTRODUÇÃO}

A expansão de áreas de pastagens cultivadas, com espécies do gênero Brachiaria no Brasil, tem se verificado em proporções, provavelmente, jamais igualadas por outras forrageiras, em qualquer outro país de clima tropical. Dentre estas espécies a que vem obtendo destaque no cenário nacional é Brachiaria brizantha cv. MG-5, com rendimentos de 10 a 18 toneladas de MS ha ${ }^{-1}$ ano $^{-1}$ e com valores nutritivos consideráveis (SOUZA, 2002).

Sabe-se que o valor nutritivo de uma planta forrageira é representado pela associação da composição bromatológica, da digestibilidade e do consumo voluntário da forragem. Dessa forma, é de grande importância o conhecimento desses, durante as diversas fases de desenvolvimento da planta, assim como a influência da época do ano e da fertilidade do solo.

A época de colheita da forragem, pelo corte ou pastejo, deve estar relacionada ao estádio de desenvolvimento da planta, consequientemente, ao seu valor nutritivo. Colheitas de forragens mais maduras implicam na obtenção de um alimento com baixa proporção de carboidratos solúveis e de baixa digestibilidade, devido ao decréscimo da relação folha/haste, que parece ser o principal fator de perda de qualidade da forragem

\footnotetext{
${ }^{1}$ Trabalho realizado na Embrapa Arroz e Feijão, Santo Antônio de Goiás.

${ }^{2}$ Mestre, Doutoranda - Departamento de Ciência dos Solo/DCS - Universidade Federal de Lavras/UFLA - Cx. P. 3037 - $37200-000$ - Lavras, MG katiazoo@hotmail.com - Bolsista CNPq

${ }_{3}^{3}$ Pesquisador da Embrapa Arroz e Feijão - Rodovia GO-462, Km 12, Zona Rural - Cx. P. 179 - 75375-000 - itamar@cnpaf.embrapa.br; belmiro@cnpaf.embrapa.br

${ }^{4}$ Doutor, Professor Titular - Departamento de Ciência dos Solo/DCS - Universidade Federal de Lavras/UFLA - Cx. P. 3037 - $37200-000$ - Lavras, MG vafaquin@ufla.br - Bolsista CNPq

${ }^{5}$ Mestre - Escola de Agronomia - Universidade Federal de Goiás/UFG - Campus Samambaia, Rodovia Goiânia, Nova Veneza, Km 0 - Cx. P. 131 74001-970 - Goiânia, GO - crissolocria@yahoo.com.br

${ }^{6}$ Mestre, Doutorando - Departamento de Ciência dos Solo/DCS - Universidade Federal de Lavras/UFLA - Cx. P. 3037 - $37200-000$ - Lavras, MG flabricio@gmail.com - Bolsista, Fapemig
} 
com a maturação (CORSI, 1990). Para Euclides et al. (1995) à medida que a planta forrageira amadurece a produção dos componentes potencialmente digestíveis (carboidratos solúveis, proteína etc.) tende a decrescer. A proporção de lignina, celulose, hemicelulose e outras frações indigestíveis aumentam, diminuindo a digestibilidade.

Com relação à variação no valor nutritivo da forragem com maior intervalo de corte, Drudi \& Favoretto (1987) encontraram teores de proteína bruta (PB) maiores nas plantas colhidas a cada 35 dias, relativamente àquelas colhidas a cada 42 dias. Em capim-Colonião, Gomide et al. (1985) revelaram uma variação de 9,8\% para $7,5 \%$ de PB quando ocorreu aumento no intervalo de corte.

O intervalo de cortes afeta ainda o potencial de rebrota e a persistência das espécies forrageiras. Geralmente, longo intervalo entre cortes leva a desvantagens como: maior deposição de material fibroso, diminuição do valor nutritivo e, conseqüentemente, do consumo. Por outro lado, cortes muitos frequientes reduzem o total de forragem produzida, diminuem as reservas das plantas e afetam o potencial de rebrota (CANTO et al., 1984). Dessa forma, deve-se procurar o ponto mais adequado para o corte buscando-se aliar a maior produção com a melhor qualidade da forragem.

O objetivo do trabalho foi avaliar o efeito de diversos intervalos de corte na produção de massa seca, altura de planta e composição químico-bromatológica da Brachiaria brizantha cv. MG-5.

\section{MATERIAL E MÉTODOS}

O experimento foi conduzido em casa de vegetação da Embrapa Arroz e Feijão, em Santo Antônio de GoiásGO. As características químicas do solo, na camada de 0$20 \mathrm{~cm}$ de profundidade, no início do experimento, foram: 6,3; Ca: 9,2 $\mathrm{cmol}_{\mathrm{c}} \mathrm{dm}^{-3}$; $\mathrm{Mg}: 1,02 \mathrm{cmol}_{\mathrm{c}} \mathrm{dm}^{-3}$; $\mathrm{Al}: 0,0 \mathrm{cmol}_{\mathrm{c}}$ $\mathrm{dm}^{-3} ; \mathrm{Al}+\mathrm{H}: 5,0 \mathrm{cmol}_{\mathrm{c}} \mathrm{dm}^{-3} ; \mathrm{CTC}: 15,3 \mathrm{cmol}_{\mathrm{c}} \mathrm{dm}^{-3} ; \mathrm{P}: 2,5 \mathrm{mg}$ $\mathrm{dm}^{-3} ; \mathrm{K}: 162 \mathrm{mg} \mathrm{dm}{ }^{-3} ; \mathrm{Cu}: 3,2 \mathrm{mg} \mathrm{dm}^{-3} ; \mathrm{Zn}: 3,0 \mathrm{mg} \mathrm{dm}^{-3}$; Mn: 57,8 $\mathrm{mg} \mathrm{dm}^{-3}$; Fe: $44 \mathrm{mg} \mathrm{dm}^{-3}$; V: $68 \%$; MO: $18 \mathrm{mg} \mathrm{dm}^{-3}$. A metodologia utilizada para a análise de solo foi da Embrapa (1997).

Foram utilizados vasos com volume de $10 \mathrm{dm}^{3}$, contendo $10 \mathrm{~kg}$ de solo (densidade do solo de $1,0 \mathrm{~g} \mathrm{dm}^{3}$ ), retirado de um ARGISSOLO VERMELHO-AMARELOeutrófico, com $30 \%$ de argila. As deficiências nutricionais foram corrigidas com aplicação $30 \mathrm{mg} \mathrm{dm}^{-3}$ de $\mathrm{P}_{2} \mathrm{O}_{5}, 8,33$ $\mathrm{mg} \mathrm{dm}^{-3}$ de $\mathrm{K}_{2} \mathrm{O}$ e $2,5 \mathrm{mg} \mathrm{dm}^{-3}$ de $\mathrm{Zn}$, utilizando como fontes: superfosfato triplo, cloreto de potássio e sulfato de zinco, respectivamente.
A semeadura nos vasos do capim MG-5, foi realizada juntamente da adubação, sendo semeadas quinze sementes por vaso. Sete dias após a emergência iniciaram-se desbastes periódicos até atingir cinco plantas por vaso.

O delineamento experimental utilizado foi inteiramente casualizado. Os tratamentos constituíram de quatro intervalos de corte (15, 20, 30 e 60 dias), com cinco repetições, totalizando 20 parcelas. Foram aplicados 100 $\mathrm{mg} \mathrm{dm}^{-3}$ de nitrogênio, utilizando como fonte o sulfato de amônio, em todas as parcelas. A adubação nitrogenada foi parcelada de acordo com os corte de avaliação da forrageira. No intervalo de corte de 15, 20, 30 e 45 dias o adubo nitrogenado foi parcelado em quatro, três, duas e uma aplicação, respectivamente.

Foram realizados quatro cortes para o intervalo de 15 dias, três corte para o de 20 dias, dois cortes para o de 30 dias e um corte para o de 60 dias. A forrageira foi cortada a uma altura de $5 \mathrm{~cm}$ do solo. O material coletado foi colocado em saco de papel identificado, pesado e colocado em estufa de ventilação forçada, com temperaturas entre 58 e $65^{\circ} \mathrm{C}$ por 72 horas, para determinação da matéria seca parcial. Após a secagem, as amostras foram moídas, passadas em peneira de $1 \mathrm{~mm}$ e armazenadas em sacos de plástico.

Foi realizada análise bromatológica para determinação da matéria seca (MS), protéina bruta (PB), fibra em detergente neutro (FDN) e fibra em detergente ácido (FDA), conforme as metodologias de Silva \& Queiroz (2002).

A análise química foi realizada para determinação das concentrações de fósforo $(\mathrm{P})$, potássio $(\mathrm{K})$, cálcio $(\mathrm{Ca})$, magnésio $(\mathrm{Mg})$, enxofre $(\mathrm{S})$, zinco $(\mathrm{Zn})$, cobre $(\mathrm{Cu})$, manganês $(\mathrm{Mn})$ e ferro $(\mathrm{Fe})$, de acordo com a metodologia de Malavolta et al. (1997).

Os dados foram analisados através do procedimento ANAVA do programa estatístico SISVAR 4.6 (FERREIRA, 1999), comparando-se as médias pelo teste de Tukey.

\section{RESULTADOS E DISCUSSÃO}

A análise de variância mostrou diferenças significativas $(\mathrm{P}<0,01)$ do efeito do intervalo de corte para a produção de massa seca e composição bromatológica do capim MG-5.

Observa-se na Tabela 1 um acréscimo na produção de massa seca da Brachiaria brizantha à medida que aumenta o intervalo de corte de 15 para 60 dias, onde as melhores respostas foram observadas no intervalo de 60 dias. A produtividade observada aos 60 dias correspondeu 
a 49,78; 55,25 e 64,01\% da obtida aos 15, 20 e 30 dias de crescimento. Mostrando que, do ponto de vista do rendimento forrageiro, a idade de corte mais apropriada para essa forrageira foi de 60 dias. Nesse período a planta está em plena fase de desenvolvimento. Costa (1995) trabalhando com capim-Marandu verificou que a melhor idade de corte visando conciliar melhor produção de massa seca e teores de PB situa-se entre 56 e 70 dias de crescimento vegetativo.

Normalmente, estudos de pastagem no campo mostram que o melhor intervalo de corte da Brachiaria brizantha para pastejo varia entre 30 e 35 dias (CORRÊA, 1999). Nesta fase, a planta apresenta altas taxas de crescimento e a qualidade da massa produzida com valores médios qualitativos ideais para a nutrição animal. $\mathrm{Na}$ pesquisa, foram realizados cortes entre 15 e 60 dias por tratar-se de um experimento em ambiente controlado de casa de vegetação, onde a forrageira foi monitorada durante todo o seu ciclo de permanência,obeservando o crescimento diferenciado à medida que a cultura permanecia em vaso, em maior período de tempo.

O mesmo comportamento da produção pode ser observado para altura de plantas, mostrando efeito significativo $(\mathrm{P}<0,01)$ crescente, ou seja, aumento no desenvolvimento da planta com o decorrer da estação de crescimento.

Comparando os teores de matéria seca (MS) nos diferentes intervalo de corte, verificou-se que com o avanço da idade das plantas, houve aumento $(\mathrm{P}<0,01)$ no teor de MS. Esses teores variaram entre 16,0 e $26,6 \%$ analisados entre 15 e 60 dias de crescimento. A planta, quando nova, apresenta altos teores de água, quanto mais próximo da sua maturidade esse teor é reduzido e ocorre um aumento nos teores de MS. De acordo com Drudi \& Favoretto (1987) à medida que prolonga o intervalo de cortes, o teor de matéria seca de forragem tende a aumentar. Resultados semelhantes também foram obtidos por Borges et al. (2002) quando trabalhavam com capim-Marandu.

$\mathrm{O}$ intervalo de corte indicou comportamento diferente para o teor de proteína bruta (PB), causando uma diminuição com o aumento de 60 dias de crescimento (Tabela 1). Os maiores teores de PB foram verificado no intervalo de corte de 15 e 20 dias, chegando a atingir 16,02 $\%$, diferenciando estatisticamente $(\mathrm{P}<0,01)$ do intervalo de 30 e 60 dias. Os elevados teores observados para PB nos tratamentos onde a forragem foi colhida em estágio vegetativo inicial, provavelmente, devem-se às elevadas percentagens de folhas e baixas porcetagens de hastes, além da conseqüente elevada relação entre folha:haste. Segundo El-Memari Neto et al. (2002) as folhas são notadamente mais digestíveis e nutricionalmente mais ricas. Castro et al. (2004) trabalhando com Brachiaria brizantha cv. Marandu com idade de corte de 28, 56, 84 e 112 dias, verificaram que os valores de $\mathrm{PB}$ diminuíram com o aumento da idade de corte.

Balsalobre et al. (2001) relatam que as maiores mudanças que ocorrem na composição das plantas forrageiras são aquelas decorrentes de sua maturidade. A maioria das espécies forrageiras sofre declínio no seu valor nutritivo com o aumento da idade, resultando da menor relação folha/haste combinada com a crescente lignificação da parede celular.

TABELA 1 - Intervalo de corte na produção de massa seca, altura de planta e composição bromatológica da Brachiaria brizantha cv. MG-5.

\begin{tabular}{ccccccc}
\hline $\begin{array}{c}\text { Intervalo de } \\
\text { corte }\end{array}$ & $\begin{array}{c}\text { Massa seca* } \\
\text { (g/vaso) }\end{array}$ & $\begin{array}{c}\text { Altura* } \\
(\mathbf{c m})\end{array}$ & $\begin{array}{c}\text { MS* } \\
(\boldsymbol{\%})\end{array}$ & $\begin{array}{c}\text { PB* } \\
(\boldsymbol{\%})\end{array}$ & $\begin{array}{c}\text { FDN* } \\
(\boldsymbol{\%})\end{array}$ & $\begin{array}{c}\text { FDA* } \\
(\boldsymbol{\%})\end{array}$ \\
\hline 15 & $17,28 \mathrm{c}$ & $29,0 \mathrm{c}$ & $16,0 \mathrm{c}$ & $16,02 \mathrm{a}$ & $57,37 \mathrm{~d}$ & $29,40 \mathrm{~d}$ \\
20 & $19,18 \mathrm{c}$ & $37,4 \mathrm{c}$ & $17,2 \mathrm{c}$ & $15,48 \mathrm{~b}$ & $60,03 \mathrm{c}$ & $31,60 \mathrm{c}$ \\
30 & $22,22 \mathrm{~b}$ & $53,4 \mathrm{~b}$ & $21,0 \mathrm{~b}$ & $12,34 \mathrm{~b}$ & $65,51 \mathrm{~b}$ & $34,00 \mathrm{~b}$ \\
60 & $34,71 \mathrm{a}$ & $76,6 \mathrm{a}$ & $26,6 \mathrm{a}$ & $8,86 \mathrm{c}$ & $70,82 \mathrm{a}$ & $36,00 \mathrm{a}$ \\
$\mathrm{CV}(\%)$ & 6,86 & 10,32 & 4,24 & 4,59 & 1,72 & 2,14 \\
\hline
\end{tabular}

Médias seguidas pela mesma letra indicam que as mesmas não diferem entre si, pelo teste de Tukey. *,**, ns significativo a 1 e $5 \%$ e não significativo. 
Contudo, o mesmo comportamento não foi observado para os teores de fibras. Houve acréscimo significativo $(P<0,01)$ do teor de FDN, conforme aumentou o intervalo de corte para 60 dias. Esse resultado é comum, devido ao acréscimo nos constituintes da parede celular. Resultados semelhantes foram constatados por Mari et al. (2004) que verificaram maiores teores de FDN, com o aumento da idade de corte.

O teor de FDN é um importante parâmetro que define a qualidade da forragem, bem como um fator que limita a capacidade ingestiva por parte dos animais. A FDN representa a fração química da forrageira que se correlaciona mais estreitamente com o consumo voluntário dos animais, sendo que valores acima de 55 a $60 \%$ correlacionam-se de maneira negativa (SOEST, 1965). No presente trabalho, os valores de FDN encontrados estiveram sempre acima do valor crítico de $55 \%$ e, portanto, o consumo voluntário das forrageiras em pastejo poderia ser limitado no caso de uma pressão de pastejo alta, que reduziria a seletividade dos bovinos. Nussio et al. (2002) relatam que forragens de elevada digestibilidade de FDN proporcionam elevado potencial de consumo de MS e, conseqüentemente, melhor produção de leite e carne.

Os teores de FDA aumentaram de forma significativa $(\mathrm{P}<0,01)$ com os intervalos de corte estudados, conforme demonstrados na Tabela 1. Os melhores teores foram observados nos intervalos de $15 \mathrm{e}$ 20 dias de crescimento. $\mathrm{O}$ teor de FDA é um fator importante quando avalia a digestibilidade de um alimento, pois à medida que aumenta os teores de FDA da forrageira, diminui a digestibilidade da MS (BRANCO, 2006).
Segundo Mari (2003) as maiores mudanças que ocorrem na composição química das plantas forrageiras são aquelas decorrentes de sua maturidade, pois, à medida que a planta forrageira amadurece, a produção dos componentes potencialmente digestíveis tende a decrescer, a proporção de lignina, celulose, hemicelulose e outras frações indigestíveis aumentam, levando à queda na digestibilidade.

As concentrações de K, Ca, Mg, S, Zn e Mn na MS não foram influenciados pelos intervalos de corte estudados (Tabela 2).

Os teores de P na MS da Brachiaria brizantha cv. MG-5 foram afetados significativamente $(\mathrm{P}<0,05)$. As maiores concentrações desse nutriente foram obtidas nos intervalos de corte de 15 e 20 dias de crescimento. Esse resultado pode ser explicado devido a maior demanda por nutrientes com o crescimento da planta. O P que chega até o sistema radicular não é suficiente para suprir suas necessidades, em função do curto intervalo de tempo em que o vegetal apresenta crescimento acelerado. Devido ao fato do tecido vegetal ser formado durante todo o tempo de crescimento, a planta continua se desenvolvendo e absorvendo nutrientes, porém em quantidade menores que a sua necessidade. Por isso, plantas jovens com mecanismo ativo de absorção podem apresentar maior concentração de nutrientes, que plantas mais velhas.

A concentração de $\mathrm{P}$ variou de $1,8 \mathrm{~g} \mathrm{~kg}^{-1}$ para os intervalos de 15 e 20 dias e de $1,6 \mathrm{~g} \mathrm{~kg}^{-1}$ de para os intervalos de 30 e 60 dias de crescimento, que segundo Malavolta (1987) são considerados normais para plantas forrageiras.

TABELA 2 - Intervalo de corte na concentração de macro e micronutrientes na Brachiaria brizantha cv. MG-5.

\begin{tabular}{|c|c|c|c|c|c|c|c|c|}
\hline $\begin{array}{l}\text { Intervalo } \\
\text { de corte }\end{array}$ & $\begin{array}{c}\mathbf{P}^{* *} \\
\left(\mathrm{~g} \mathrm{~kg}^{-1}\right)\end{array}$ & $\begin{array}{c}\mathrm{K}^{\mathrm{ns}} \\
\left(\mathrm{g} \mathrm{kg}^{-1}\right)\end{array}$ & $\begin{array}{c}\mathrm{Ca}^{\mathrm{ns}} \\
\left(\mathrm{g} \mathrm{kg}^{-1}\right)\end{array}$ & $\begin{array}{c}\mathrm{Mg}^{\mathrm{ns}} \\
\left(\mathrm{g} \mathrm{kg}^{-1}\right)\end{array}$ & $\begin{array}{c}\mathrm{Zn}^{\text {ns }} \\
\left(\mathrm{mg} \mathrm{kg}^{-1}\right)\end{array}$ & $\begin{array}{c}\mathrm{Cu}^{* *} \\
\left(\mathrm{mg} \mathrm{kg}^{-1}\right)\end{array}$ & $\begin{array}{c}\mathrm{Mn}^{\mathrm{ns}} \\
\left(\mathrm{mg} \mathrm{kg}^{-1}\right)\end{array}$ & $\begin{array}{c}\mathrm{Fe}^{* * *} \\
\left(\mathrm{mg} \mathrm{kg}^{-1}\right)\end{array}$ \\
\hline 15 & $1,8 \mathrm{a}$ & $2,6 \mathrm{a}$ & $5,10 \mathrm{a}$ & $3,01 \mathrm{a}$ & $1,90 \mathrm{a}$ & $5,8 \mathrm{a}$ & $12,08 \mathrm{a}$ & $11,58 \mathrm{a}$ \\
\hline 20 & $1,8 \mathrm{a}$ & $2,6 \mathrm{a}$ & $5,00 \mathrm{a}$ & $3,02 \mathrm{a}$ & $1,88 \mathrm{a}$ & $5,8 \mathrm{a}$ & $10,30 \mathrm{a}$ & $10,50 \mathrm{a}$ \\
\hline 30 & $1,6 \mathrm{~b}$ & $2,5 \mathrm{a}$ & $4,82 \mathrm{a}$ & $3,22 \mathrm{a}$ & $1,70 \mathrm{a}$ & $4,9 \mathrm{~b}$ & $10,60 \mathrm{a}$ & $8,28 \mathrm{~b}$ \\
\hline 60 & $1,6 \mathrm{~b}$ & $2,2 \mathrm{a}$ & $4,27 \mathrm{a}$ & $3,10 \mathrm{a}$ & $1,55 \mathrm{a}$ & $4,2 \mathrm{~b}$ & $11,12 \mathrm{a}$ & $7,00 \mathrm{~b}$ \\
\hline CV $(\%)$ & 13,5 & 12,37 & 14,30 & 20,71 & 11,56 & 12,08 & 12,94 & 16,33 \\
\hline
\end{tabular}

Médias seguidas pela mesma letra indicam que as mesmas não diferem entre si, pelo teste de Tukey. *, **, ns significativo a 1 e $5 \%$ e não significativo. 
De acordo com o NRC (2001), a concentração de P na MS, recomendada para bovinos de corte é de 0,5 a $2,5 \mathrm{~g} \mathrm{~kg}^{-1}$. Assim, os valores encontrados neste estudo, estão entre os recomendados pelo NRC.

$\mathrm{O}$ intervalo de corte influenciou significativo $(\mathrm{P}<0,05)$ na concentração de $\mathrm{Cu}$. Observa-se na Tabela 2 que houve uma queda na concentração de $\mathrm{Cu}$, à medida que aumenta o intervalo de corte para 60 dias de crescimento. As concentrações deste nutriente variaram entre 4,2 a 5,8 mg $\mathrm{kg}^{-1}$. Esses níveis de $\mathrm{Cu}$ encontrados na Brachiaria brizantha $\mathrm{cv}$. MG-5 podem ser considerados baixos, pois segundo Malavolta (1987), os teores adequados para as braquiárias na MS é $6,0 \mathrm{mg} \mathrm{kg}^{-1}$. Esses resultados podem ser atribuídos ao pH do solo que se encontrava em 6,3. A absorção de $\mathrm{Cu}$ é prejudicada em $\mathrm{pH}$ superior a 5,0; porque esse nutriente fica retido firmemente nos colóides do solo, havendo redução da sua disponibilidade, para a planta. Primavesi et al. (2006) trabalhando com doses de nitrogênio no capim-Marandu, verificaram concentrações mais altas de $\mathrm{Cu}$, variando de 7 a $10 \mathrm{mg} \mathrm{kg}^{-1}$.

Houve efeito significativo $(\mathrm{P}<0,05)$ do intervalo de corte para concentrações de $\mathrm{Fe}$, ocorrendo uma redução na absorção, com o aumento dos intervalos de corte estudados. A planta nova usou todo o seu potencial na absorção destes nutrientes. À medida que a planta desenvolvia maior quantidade de massa seca estava sendo sintetizada. A planta, de acordo com as condições do ambiente, intensificou seu crescimento, e o fluxo de nutrientes permaneceu constante ocorrendo uma diluição no tecido da planta.

As concentrações de Fe ficaram entre 115,8 a 70,0 $\mathrm{mg} \mathrm{kg}^{-1}$ para os intervalos de 15 e 60 dias de crescimento. A concentração desse nutriente observada na análise do solo, apresentava níveis suficientes para suprir a necessidade da cultura. Contudo, os níveis de $\mathrm{Fe}$ encontrados na forrageira podem ser considerados baixos, que segundo Malavolta (1987) os teores adequados para as braquiárias na MS é 180 a $250 \mathrm{mg} \mathrm{kg}^{-1}$. A absorção de $\mathrm{Fe}$ também é prejudicada em solos alcalinos ou solos corrigidos que receberam pesadas doses de calcário.

De acordo McDowell (1999), o requerimento estimado de ferro para ruminante adulto encontra-se entre 30 a $60 \mathrm{mg} \mathrm{kg}^{-1}$ e para bezerros este requerimento é de $100 \mathrm{mg} \mathrm{kg}^{-1}$ mostrando que a exigência para o animal jovem é maior que para o adulto. A Brachiaria brizantha cv. MG-5 atenderia a essa exigência, mesmo nos intervalos de corte maiores. Com o menor intervalo a concentração de Fe apresentou valores superiores, porém abaixo do nível considerado tóxico para os animais que é de $1000 \mathrm{mg} \mathrm{kg}^{-1}$.

\section{CONCLUSÕES}

O aumento no intervalo de corte na Brachiaria brizantha cv. M-5 determina acréscimos na produção de massa seca e teores de FDN e FDA. Contudo, o inverso pode ser observado no teor de $\mathrm{PB}$ e concentrações de $\mathrm{P}, \mathrm{Cu}$ e Fe.

\section{REFERÊNCIAS BIBLIOGRAFICAS}

BALSALOBRE, M. A. A.; NUSSIO, L. G.; MARTHA JÚNIOR, G. B. Controle de perdas na produção de silagem de gramíneas tropicais. In: SIMPÓSIO SOBRE MANEJO DA PASTAGEM, 2001, Piracicaba. Anais... Piracicaba: FEALQ, 2001. p. 890-911.

BORGES, A. L. C. C.; RABELO, L. S.; GONÇALVES, L. C. Avaliação da Brachiaria brizantha, cv. Marandu em oito idades de corte em Igarapé-MG: teores de matéria seca e relação folha: haste. In: REUNIÃO ANUAL DA SOCIEDADE BRASILEIRA DE ZOOTECNIA, 39., 2002, Recife. Anais... Recife: SBZ, 2002. CD-ROM.

BRANCO, A. F. Caracterização de alimentos para ruminantes. 2006. Disponível em: <http:// www.potasal.com.br>. Acesso em: 10 fev. 2006.

CANTO, A. C.; TEIXEIRA, L. B.; ITALIANO, E. E. Capineiras de corte para a região de Manaus, Amazonas. Manaus: Embrapa-UEPAE, 1984. 29 p.

CASTRO, G. H. F.; GRAÇA, D. S.; GONÇALVES, L. C.; BORGES, I.; POSSAS, F. P.; JAYME, C. G.; NOGUEIRA, U. T.; RODRIGUEZ, N. M.; BORGES, A. L. C.; SALIBA, E. S. Degradabilidade in situ da matéria seca e proteína bruta da Brachiaria brizantha cv. Marandu em quatro diferentes idades de corte. In: REUNIÃO ANUAL DA SOCIEDADE BRASILEIRA DE ZOOTECNIA, 41., 2004, Campo Grande. Anais... Campo Grande: SBZ, 2004. CD-ROM.

CORRÊA, L. A. Produção de gado de corte em pastagens adubadas. In: SIMPÓSIO SOBRE PRODUÇÃO DE BOVINOS DE CORTE, 1., 1999, Goiânia. Anais... Goiânia: CBNA, 1999. p. 81-94.

CORSI, M. Produção e qualidade de forragens tropicais. In: SIMPÓSIO SOBRE MANEJO DA PASTAGEM, 1990, Piracicaba. Anais... Piracicaba: FEALQ, 1990. p. 69-85.

COSTA, N. L. Curva de crescimento e composição química da Brachiaria brizantha cv. Marandu em Rondônia. In: REUNIÃO ANUAL DA SOCIEDADE BRASILEIRA DE ZOOTECNIA, 32., 1995, Brasília, DF. Anais... Brasília, DF: SBZ, 1995. p. 38-40. 
DRUDI, A.; FAVORETTO, V. Influência da freqüência, época e altura do corte na produção e na composição química do capim-andropógon. Pesquisa Agropecuária Brasileira, Brasília, v. 22, n. 12, p. 1287-1292, 1987.

EL-MEMARI NETO, A. C.; ZEOULA, L. M.; CECATO, U. Avaliação produtiva e química da Brachiaria brizantha no inverno e primavera. In: REUNIÃO ANUAL DA SOCIEDADE BRASILEIRA DE ZOOTECNIA, 39., 2002, Recife. Anais... Recife: SBZ, 2002. CD-ROM.

EMPRESA BRASILEIRA DE PESQUISA AGROPECUÁRIA. Centro Nacional de Pesquisa de Solos. Ministério da Agricultura e do Abastecimento. Manual de métodos de análise de solo. 2. ed. rev. e atual. Rio de Janeiro: Embrapa-CNPS, 1997. 212 p.

EUCLIDES, V. P. B.; MACEDO, M. C. M.; VALLE, L. C. S Avaliação de acessos de Panicum maximum sob pastejo. Campo grande: Embrapa-CNPGC, 1995. 7 p.

FERREIRA, D. F. SISVAR - Sistema de análise de variância para dados balanceados. Lavras: UFLA, 1999.

GOMIDE, J. A.; OBEID, J. A.; QUEIROZ, D. S. Freqüência de cortes,espaçamento entre fileiras e adubação de capimcolonião (Panicum maximum Jacqes) e capim-jaraguá (Hyparrenia rufa, (Nees, Stapf). Revista Brasileira de Zootecnia, Viçosa, v. 14, n. 3, p. 326-337, 1985.

MALAVOLTA, E. Manual de calagem e adubação das principais culturas. São Paulo: Ceres, 1987. 496 p.

MALAVOLTA, E.; VITTI, G. C.; OLIVEIRA, S. A. Avaliação do estado nutricional das plantas: princípios e aplicações. 2. ed. Piracicaba: Associação Brasileira da Potassa e do Fosfato, 1997. 319 p.

MARI, L. J. Intervalo entre corte em capim-Marandu (Brachiaria brizantha (Hochst. ex A. Rich.) Stapf cv. Marandu): produção, valor nutritivo e perdas associadas a fermentação da silagem. 2003. 138 f. Dissertação (Mestrado) - Escola Superior de Agricultura "Luiz de
Queiroz”, Universidade de São Paulo, Piracicaba, 2003.

MARI, L. J.; NUSSIO, L. G.; SCHMIDT, P.; PAZIANI, S. F.; RIBEIRO, J. L.; ZOPOLLATTO, M.; JUNQUEIRA, M. C.; LOURES, R. S.; PEDROSO, A. F. Magnitude das alterações na composição morfológica e valor nutritivo do capimMarandu (Brachiaria brizantha, Stapf. cv. Marandu) mantido a intervalos fixos entre cortes. In: REUNIÃO ANUAL DA SOCIEDADE BRASILEIRA DE ZOOTECNIA, 41., 2004, Campo Grande. Anais... Campo Grande: SBZ, 2004. CD-ROM.

McDOWELL, L. R. Minerais para ruminantes sob pastejo em regiões tropicais, enfatizando o Brasil. 3. ed. Florida: University of Florida, 1999. 89 p.

NATIONAL RESEARCH COUNCIL. Nutrient requeriments of dairy cattle. 7 . ed. Washington: National Academy, 2001. 381 p.

NUSSIO, G. L.; CAMPOS, F. P.; PAZIANI, S. F.; SANTOS, F. A. P. Volumosos suplementares: estratégias de decisão e utilização. In: FORRAGICULTURA E PASTAGENS: TEMAS EM EVIDÊNCIA, 2002, Lavras. Anais... Lavras: UFLA, 2002. p. 193-232.

PRIMAVESI, A. C.; PRIMAVESI, O.; CORRÊA, L. A.; SILVA, A. G.; CANTARELLA, H. Nutrientes na fitomassa de capimmarandu em função de fontes e doses de nitrogênio. Revista Ciência e Agrotecnologia, Lavras, v. 30, n. 3, p. 562-568, 2006.

SILVA, D. J.; QUEIROZ, A. C. Análise de alimentos: métodos químicos e biológicos. 3. ed. Viçosa: UFV, 2002. $235 \mathrm{p}$.

SOEST, P. J. van. Symposium on factors influencing the voluntary intake of herbage by ruminants: voluntary intake relation to chemical composition and digestibility. Journal of Animal Science, Champaign, v. 24, n. 3, p. 834-844, 1965.

SOUZA, F. H. D. As sementes de espécies forrageiras do gênero Brachiaria no Brasil Central. In: PAULINO, V. T. A Brachiaria no novo século. 2. ed. Nova Odessa: Instituto de Zootecnia, 2002. 